\title{
Gradient-driven flux-tube simulations of ion temperature gradient turbulence close to the non-linear threshold
}

\author{
A.G. Peeters ${ }^{1}$, F. Rath ${ }^{1}$, R. Buchholz ${ }^{1}$, Y. Camenen ${ }^{2}$, J. Candy ${ }^{3}$, \\ F.J. Casson ${ }^{4}$, S.R. Grosshauser ${ }^{1}$, W.A. Hornsby ${ }^{5}$, D. Strintzi ${ }^{1}$, A. Weikl ${ }^{1}$ \\ 1 University of Bayreuth, Physics department, Universitätsstrasse 30 Bayreuth, Germany \\ ${ }^{2}$ Aix Marseille Univ, CNRS, PIIM, UMR 7345, Marseille, France \\ 3 General Atomics, PO Box 85608, San Diego, CA 92186-5608, USA \\ 4 CCFE, Culham Science Centre, Abingdon OX14 3DB, Oxon, England and \\ ${ }^{5}$ Max Planck Institut für Plasmaphysik, Boltzmannstrasse 285748 Garching, Germany
}

\begin{abstract}
It is shown that ITG turbulence close to the threshold exhibits a long time behaviour, with smaller heat fluxes at later times. This reduction is connected with the slow growth of long wave length zonal flows and, consequently, the numerical dissipation on these flows must be sufficient small. Close to the nonlinear threshold for turbulence generation, a relatively small dissipation can maintain a turbulent state with a sizeable heat flux, through the damping of the zonal flow. Lowering the dissipation causes the turbulence, for temperature gradients close to the threshold, to be subdued. The heat flux then does not go smoothly to zero when the threshold is approached from above. Rather, a finite minimum heat flux is obtained below which no fully developed turbulent state exists. The threshold value of the temperature gradient length at which this finite heat flux is obtained is up to $30 \%$ larger compared with the threshold value obtained by extrapolating the heat flux to zero, and the cyclone base case is found to be nonlinearly stable. Transport is subdued when a fully developed staircase structure in the ExB shearing rate forms. Just above the threshold, an incomplete staircase develops, and transport is mediated by avalanche structures which propagate through the marginally stable regions.
\end{abstract}

\section{INTRODUCTION}

Energy confinement in fusion plasmas is determined by turbulent transport, with the Ion Temperature Gradient (ITG) mode expected to dominate under reactor conditions. ITG turbulence arises through a small scale (ion Larmor radius) instability, which is dominantly driven by the ion temperature gradient, and has a threshold in the gradient length $\left(R / L_{T l i n}\right)$, above which the mode is unstable. The non-linear threshold $\left(R / L_{\text {Tdim }}\right)$, above which a stationary turbulent state develops, however, is larger ('up-shifted') compared with the linear threshold, a phenomenon known as the Dimits shift [1]. ITG turbulence, through nonlinear interactions, generates zonal flows which through shear stabilization regulate the turbulence [2-4]. For gradient lengths $R / L_{\text {Tlin }}<R / L_{T}<$ $R / L_{\text {Tdim }}$, the shear flow quenches the turbulence.

In the original work [1], the non-linear threshold $R / L_{\text {Tdim }}$ is obtained by extrapolating the heat flux of several simulations with gradient lengths $R / L_{T}>$ $R / L_{\text {Tdim }}$, to obtain the gradient length at which the heat flux goes to zero. Recently, the non-linear threshold for turbulence generation has been revisited $[5,6]$. For gradient-driven simulations, it was shown that the heat flux does not go smoothly to zero when the threshold is approached from above. Rather, the heat flux has a finite value at a non-linear threshold $R / L_{T c}>R / L_{\text {Tdim }}$, below which no stationary turbulent state is obtained. In this paper the gradient-driven case is investigated in more detail.

\section{NUMERICAL SET-UP}

The plasma parameters for the study in this paper are those of the cyclone base case: safety factor $q=1.4$, magnetic shear $\hat{s}=0.78$, inverse aspect ratio $\epsilon=0.19$, density gradient $R / L_{n}=2.2$, and electron to ion temperature ratio $T_{e} / T_{i}=1$. The temperature gradient is scanned to determine the threshold. In order to compare the results with the original work, the electro-static limit with the adiabatic electron response is investigated, and collisions are neglected. Various resolutions have been used in the numerical simulations and are given in Table I. The maximum of the velocity grid, is three times the thermal velocity for both the parallel as well as the perpendicular velocity, in all cases.

The simulations are performed with the flux tube version of the non-linear gyro-kinetic code GKW [7]. The temperature gradient is prescribed, and periodic boundary conditions are used in the plane perpendicular to the magnetic field. Different from Refs. [5, 6], in which the radial direction is treated with finite differences, the simulations in this paper use a spectral representation in both the radial as well as the bi-normal direction. Details of the model equations and implementation are given in Ref. [7]. Since numerical dissipation turns out to be important, some details of the implementation are given below.

In the turbulent state, the entropy production due to the heat flux down the temperature gradient is balanced by dissipation [8]. Furthermore, in Eulerian codes like GKW, grid scale dissipation plays an essential role in ob- 
taining numerical stability. Therefore, dissipation must be applied to obtain a stationary turbulent state. However, if the applied dissipation is not linked with a physical process, like collisions, the predicted turbulent fluxes should not be affected by its arbitrary magnitude. Indeed, it has been shown that the fluxes are insensitive to the magnitude of the applied dissipation [9] for simulations well above the nonlinear threshold, provided the dissipation is sufficiently small. The precise form of the dissipation on the scale of the grid, then does not affect macroscopic quantities like the heat flux.

The spectral version of GKW has two convective derivatives

$$
\frac{\partial f}{\partial t}+v_{\|} \mathbf{b} \cdot \nabla f-\frac{\mu}{m} \mathbf{b} \cdot \nabla B \frac{\partial f}{\partial v_{\|}}+\ldots=0,
$$

modelling the motion along the magnetic field (second term) and the particle trapping in the magnetic well (third term). In the equation above, $f$ is the perturbed distribution, $v_{\|}$the parallel velocity, $\mathbf{b}$ the unit vector in the direction of the magnetic field, $B$ the magnetic field strength, and $\mu$ the magnetic moment. These convective derivatives require dissipation in order to obtain numerical stability. Both are treated using a five point stencil, with the motion along the magnetic field represented using

$$
\begin{aligned}
& v_{\|} \mathbf{b} \cdot \nabla f \rightarrow F_{i} v_{\|} \frac{f_{i-2}-8 f_{i-1}+8 f_{i+1}-f_{i+2}}{12 \Delta s} \\
& +D F_{i} v_{d} \frac{f_{i-2}-4 f_{i-1}+6 f_{i}-4 f_{i+1}+f_{i+2}}{12 \Delta s},
\end{aligned}
$$

where $i$ denotes the grid point considered, $s$ is the parallel coordinate in straight field line Hamada coordinates, and $\Delta s$ is the distance between the grid points. The parallel coordinate is normalized such that one poloidal turn corresponds to an interval $s=\left[\begin{array}{ll}-0.5 & 0.5\end{array}\right]$ and, consequently, $\Delta s=1 / N_{s}$, where $N_{s}$ is the number of grid points along the magnetic field. The quantity $F=B^{s} / B$, where $B^{s}=\mathbf{B} \cdot \nabla s$ is the contra-variant component of the magnetic field can, for circular geometry, be approximated by $F \approx 1 / 2 \pi q R$. The first term in Eq. (2) is the approximation of the convective derivative and is fourth order accurate in space, while the second term, proportional to $D$, represents the numerical dissipation, which satisfies

$$
\begin{aligned}
& D v_{d} F_{i} \frac{f_{i-2}-4 f_{i-1}+6 f_{i}-4 f_{i+1}+f_{i+2}}{12 \Delta s} \\
= & \frac{D(\Delta s)^{3} v_{d} F}{12} \frac{\partial^{4} f}{\partial s^{4}}+\mathcal{O}\left(\Delta s^{2}\right) .
\end{aligned}
$$

When the velocity $v_{d}$ is set equal to the absolute value of parallel velocity $v_{d}=\left|v_{\|}\right|$, the scheme is equivalent to a third order upwind scheme if $D=1$. However, $v_{d}$ can also be taken to be a constant $v_{d}=\sqrt{3} v_{t h}$, where $v_{t h}=\sqrt{2 T / m_{i}}$ is the thermal velocity. In the latter case the dissipation has no velocity dependence. For $D \approx 1$, a value usually required for stability, the second term is of equal magnitude compared with the first term, for perturbations with wavelengths of the order of the grid size. The latter perturbations are then effectively damped. Increasing the number of grid points by a factor two, does not change the damping at the (finer) grid scale, but reduces the damping at fixed wavelengths by a factor eight.

For unstable modes, the dissipation has little influence as long as the damping of the mode through the dissipation is much smaller than its growth rate. This condition can easily be satisfied for the modes that generate the turbulent fluxes, but not for the zonal mode, since it has zero growth rate. Indeed, when investigating the effect of collisions, Ref. [10] found that the ion heat flux depends on the collision frequency, even if the instabilities are collisionless, through the collisional damping of the zonal mode. The largest influence of the dissipation is, therefore, expected to occur through the damping of the zonal mode. In the inhomogeneous magnetic field the ExB velocity, associated with the zonal mode, is not divergence free. A Pfirsch Schlüter type dependence in the perturbed distribution then arises, which has a spatial dependence $f=A \cos (2 \pi s)$. Using the equations above, the dissipation then yields a typical damping rate $\gamma_{d}^{(4)}\left(N_{s}\right)=-D(\sqrt{3} / 12 q)\left(2 \pi / N_{s}\right)^{3}$, where $\gamma_{d}^{(4)}$, and all other time-scales in this paper, are normalized with $v_{t h} / R$. For $D=1$ this corresponds to $\gamma_{d}^{(4)}(16)=-6.2 \cdot 10^{-3}$, and $\gamma_{d}^{(4)}(32)=-7.8 \cdot 10^{-4}$. These damping rates are much smaller than the ITG growth rate of the cyclone base case $\gamma=0.188(\hat{s}-\alpha$ geometry, with $R / L_{T}=6.9, k_{y} \rho=0.4$, and $k_{y} \approx n q / r$ with $n$ the toroidal mode number, and $r$ the minor radius of the surface). They are, however, not necessarily small compared with the the collisional damping rate of zonal mode under experimentally relevant conditions. The latter damping rate is $\gamma_{C} \approx-R \nu_{i i} /\left(2.7 \epsilon v_{t h}\right)=-1 \cdot 10^{-3} R n_{19} T_{k}^{-2}$ [11], where $\nu_{i i}$ is the ion-ion collision frequency, $n_{19}$ is the particle density in $10^{19} \mathrm{~m}^{-3}, T_{k}$ is the temperature in $\mathrm{keV}$, and in the last step a Deuterium plasma with $\epsilon=0.19$ has been assumed. In many current experiments, and certainly in a reactor, the collisional damping rate can be considerably smaller than the dissipation at a resolution $N_{s} \approx 16$. The DIII-D discharge \# 81499, from which the original cyclone base case parameters were obtained, for instance, has a collisional damping rate $\gamma_{C}=1.9 \cdot 10^{-3}$ (with $n_{19}=4.5, T_{k}=2$ and $R=1.7$ ).

The same numerical scheme is used for both the parallel motion along the magnetic field and the trapping term. In order to estimate the damping of the zonal flow due to the dissipation added in the trapping term a scale length in velocity space equal to the size of the trapped domain $\left(\sqrt{2 \epsilon} v_{t h}\right)$ is assumed. Indeed, using this estimate for the collision induced diffusion in velocity space, yields a collisional damping rate $\gamma_{C}=-R \nu_{i i} / 2 \epsilon v_{t h}$, in close agreement with the results of Ref. [11]. Using this typical scale, the damping rate can be estimated to be $\gamma_{v}\left(N_{v_{\|}}\right)=$ 
$-D_{v_{\|}} /(3 q \epsilon)\left(v_{\|}^{\max } / N_{v_{\|}} v_{t h}\right)^{3}$. For $v_{\|}^{\max }=3 v_{t h}, D_{v_{\|}}=1$, this yields $\gamma_{v}(32)=-1 \cdot 10^{-3}$ and $\gamma_{v}(64)=-1.3 \cdot 10^{-4}$. The fourth order scheme was chosen in the original implementation of GKW [7] because of its high accuracy and low dissipation. The results presented in this paper will, however, show that the dissipation is not for all cases sufficiently small. Therefore, additionally a sixth order scheme has been implemented for the derivatives along the magnetic field of the zonal mode (all other modes as well as the velocity space derivative are unchanged)

$v_{\|} \mathbf{b} \cdot \nabla f=$

$v_{\| i} F_{i} \frac{-f_{i-3}+9 f_{i-2}-45 f_{i-1}+45 f_{i+1}-9 f_{i+2}+f_{i+3}}{60 \Delta s}-$

$v_{d} F_{i} \frac{f_{i-3}-6 f_{i-2}+15 f_{i-1}-20 f_{i}+15 f_{i+1}-6 f_{i+2}+f_{i+4}}{60 \Delta s}$

In this case the dissipation given by the last term on the right hand side is approximately

$$
\approx \frac{v_{d} F(\Delta s)^{5}}{60} \frac{\partial^{6} f}{\partial s^{6}}+\mathcal{O}\left(\Delta s^{2}\right)
$$

yielding a damping rate $\gamma_{d}^{(6)}\left(N_{s}\right)=-\sqrt{3} D / 60 q\left(2 \pi / N_{s}\right)^{5}$, or $\gamma_{d}^{(6)}(16)=-1.9 \cdot 10^{-4}$ for $D=1$.

Additional to the dissipation in the convective terms, a damping of high wave vector modes can be applied

$$
\frac{\partial f}{\partial t}=-D_{y}\left(\frac{k_{y}}{k_{y}^{\max }}\right)^{4} f-D_{x}\left(\frac{k_{x}}{k_{x}^{\max }}\right)^{4} f,
$$

where $k_{x}\left(k_{y}\right)$ is the radial (bi-normal) wave vector and $k_{x}^{\max }\left(k_{y}^{\max }\right)$ is the maximum value of the radial (binormal) wave vector kept in the simulations. Note that all dissipation is applied only in the terms that involve the distribution function. Dissipation of the field terms in general leads to a numerical instability [12].

\section{MODERATE RESOLUTION AND TIME INTERVAL SIMULATIONS}

Fig. 1 shows the heat conduction coefficients in gyroBohm units $\rho^{2} v_{t h} / R$, where $\rho=m_{i} v_{t h} / e B$ is the ion Larmor radius, $v_{t h}=\sqrt{2 T / m_{i}}$ is the thermal velocity, $T$ is the background temperature, $e$ is the unit charge, and $R$ is the major radius. Note that the thermal velocity and Larmor radius are $\sqrt{2}$ larger than the often used sound speed $c_{s}=\sqrt{T_{e} / m_{i}}$ and its corresponding Larmor radius $\rho_{s}=m_{i} c_{s} / e B$. The results are obtained for a standard resolution (case $\mathrm{S}$ of Table I), and a total simulation time interval of $2400 R / v_{t h}$. Two different geometry models have been used: the $\hat{s}-\alpha$ geometry and a circular geometry in which all orders of the inverse aspect ratio $(\epsilon)$ are kept [13]. The dashed lines in the graph represent the

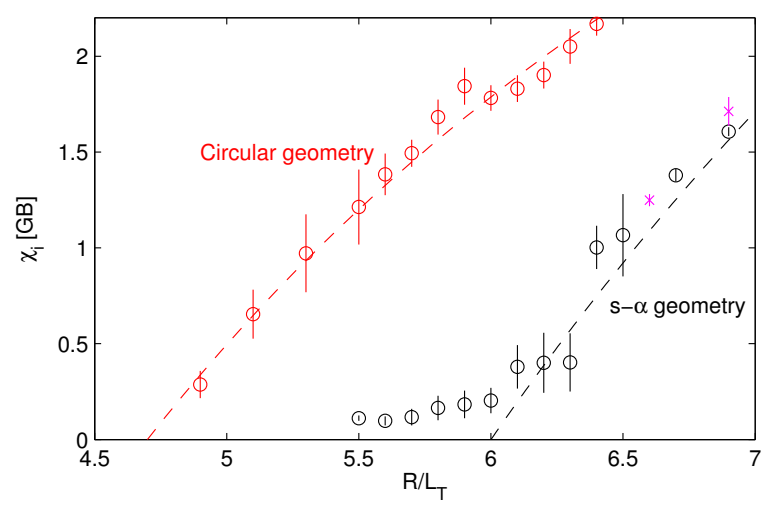

FIG. 1. Heat conduction coefficient $\chi$ in units $\rho^{2} v_{t h} / R$ as a function of the normalized gradient length $R / L_{T}$, for both the $\hat{s}-\alpha$ (colour online: black) as well as the circular geometry (colour online: red). Crosses (colour online: magenta) represent the results of runs with the GYRO code and are discussed in Section V.

Dimits fit, adjusted for the different normalization used in this paper,

$$
\chi=12\left(1-\frac{6}{R / L_{T}}\right)
$$

valid for the $\hat{s}-\alpha$ geometry, and a similar fit

$$
\chi=8.25\left(1-\frac{4.7}{R / L_{T}}\right)
$$

for the circular geometry. The latter is obtained by fitting the data in the graph. Note that the non-linear threshold in the gradient length is smaller for the circular geometry compared with the $\hat{s}-\alpha$ geometry. The error bars in the graph, and in all other figures in this paper, represent the statistical error only, and have been obtained by dividing the stationary phase into three time windows, and by taking the standard deviation of the mean values obtained for each of these windows. The heat conduction in these simulations goes, more or less, smoothly to zero when the threshold is approached from above. Although the error bars get larger close to the threshold of the $\hat{s}-\alpha$ model, the results agree within the error bars with the Dimits fit $\left(\chi_{f i t}\right)$, and the value well above the threshold at $R / L_{T}=6.9$ of $\chi=1.61$ is in excellent agreement $\left(\chi_{\text {fit }}=1.56\right)$. A benchmark for larger values of the gradient length can be found in Ref. [14].

Below the non-linear threshold $\left(R / L_{T \text { dim }}=6\right)$ of the $\hat{s}-\alpha$ geometry, a small but finite heat flux is obtained. This region of parameter space is investigated in more detail below. Fig. 2 shows the time traces of three simulations with $R / L_{T}=5.8$ : The simulation from which the data in Fig. 1 is taken (case $\mathrm{S}$ in Table I, red curve in the online version), a simulation in which the velocity space resolution as well as $N_{s}$ have been doubled (case D in Table I, blue curve in the online version), and a simulation 
TABLE I. The Resolutions used in this paper: Number of toroidal modes $N_{m}$, number of radial modes $N_{x}$, number of grid points along the magnetic field $N_{s}$, number of parallel velocity grid points $N_{v_{\|}}$, number of magnetic moment grid points $N_{\mu}$, dissipation coefficient used in convection along the magnetic field $D$, the velocity in the dissipation scheme $v_{d}$, dissipation coefficient used in the trapping term $D_{v_{\|}}$, damping coefficient of radial modes $D_{x}$, damping coefficient of toroidal modes $D_{y}$, Order of the scheme used for the zonal mode, maximum poloidal wave vector $k_{y} \rho^{\text {max }}$, and maximum radial wave vector $k_{x} \rho^{\text {max }}$

\begin{tabular}{|l|c|c|c|c|c|c|c|c|c|c|c|c|c|}
\hline Case & $N_{m}$ & $N_{x}$ & $N_{s}$ & $N_{v_{\|}}$ & $N_{\mu}$ & $D$ & $v_{d}$ & $D_{v_{\|}}$ & $D_{x}$ & $D_{y}$ & Order & $k_{y} \rho^{\max }$ & $k_{x} \rho^{\max }$ \\
\hline Standard resolution (S) & 21 & 83 & 16 & 64 & 9 & 1 & $\sqrt{3} v_{t h}$ & 0.2 & 0.1 & 0.1 & 4 & 1.4 & 2.1 \\
Standard resolution with 6th order (S6) & 21 & 83 & 16 & 64 & 9 & 1 & $\left|v_{\|}\right|$ & 0.2 & 0.1 & 0.1 & 6 & 1.4 & 2.1 \\
Double resolution (D) & 21 & 83 & 32 & 128 & 18 & 1 & $\sqrt{3} v_{t h}$ & 0.2 & 0.1 & 0.1 & 4 & 1.4 & 2.1 \\
Double resolution high dissipation (DD) & 21 & 83 & 32 & 128 & 18 & 8 & $\sqrt{3} v_{t h}$ & 1.6 & 0.1 & 0.1 & 4 & 1.4 & 2.1 \\
Low dissipation high perpendicular resolution (LH) & 41 & 167 & 32 & 64 & 9 & 1 & $\sqrt{3} v_{t h}$ & 0.03 & 0.1 & 0.1 & 4 & 2.0 & 4.0 \\
Low dissipation standard resolution (LS) & 21 & 83 & 32 & 192 & 9 & 1 & $\sqrt{3} v_{t h}$ & 1 & 0.1 & 0.1 & 4 & 1.4 & 2.1 \\
Benchmark (G6) & 21 & 83 & 24 & 64 & 24 & 1 & $\left|v_{\|}\right|$ & 0.2 & 0.1 & 0.1 & 6 & 1.4 & 2.1 \\
\hline
\end{tabular}
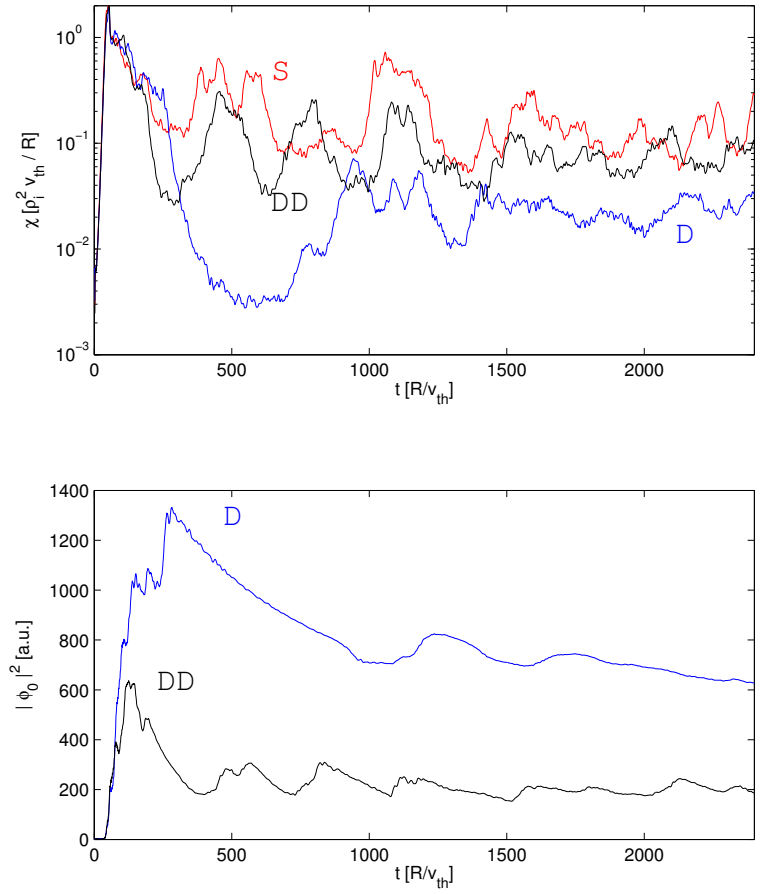

FIG. 2. Top: time traces of the heat conduction coefficient for the $\hat{s}-\alpha$ geometry with $R / L_{T}=5.8$ and different resolution / dissipation labelled according to Table I. Bottom: the squared potential of the $k_{y}=0$ mode summed over all radial modes, as a function of normalized time.

in which both the resolution as well as the dissipation coefficients have been increased (case DD in Table I, black curve in the online version). In the latter case the dissipation connected with the convective terms is the same as the original (case S) simulation. Taking the average over the time interval [180-2400], the simulations yield:
$\chi_{S}=0.19, \chi_{D}=0.034$, and $\chi_{D D}=0.089$. Although, the resolution of the numerical solution plays a role, the results above show that the finite heat flux below $R / L_{T}=6$ also depends strongly on the applied dissipation, with higher dissipation leading to higher heat fluxes. This, of course, means the results are non-physical since the dissipation should not affect macroscopic quantities like the heat flux. Some insight into the reason of the finite heat flux below $R / L_{T}=6$ can be obtained from the time trace of $\sum_{k_{x}}\left|\phi_{0, k_{x}}\right|^{2}$, where $\phi\left(0, k_{x}\right)$ is the potential perturbation of the $k_{y}=0$ (zonal) mode, and the sum is taken over all radial modes. This time trace is shown in the bottom graph of Fig. 2. Initially, the turbulence spins up a zonal flow which, when the turbulence is reduced through ExB shearing, starts to decay. A decay rate can be estimated from the latter phase to be $\gamma=-5.5 \cdot 10^{-3}$ for the high dissipation case (DD), and $\gamma=-8.2 \cdot 10^{-4}$ for the low dissipation case (D). These decay rates are in good agreement with the estimates based on the dissipation given above $\left(\gamma_{d}^{(4)}=-6.2 \cdot 10^{-3}\right.$ and $\gamma_{d}^{(4)}=-7.8 \cdot 10^{-4}$, respectively). The decay of the zonal mode is, therefore, attributed to the dissipation in the convection term. As the zonal mode amplitude decays, and falls below a certain value, temporal limited phases of higher turbulent activity reappear, which in turn drive the zonal mode, leading to an increases in its amplitude. This behaviour is similar to the behaviour observed when collisions are included [10]. When averaged over a long time interval, a finite heat flux is obtained which, however, is linked with the dissipative damping and is, therefore, considered non-physical. Investigating the simulations shown in Fig. 1 it is, furthermore, found that for $R / L_{T} \leq 6.3$ the heat flux exhibits temporal limited quiet phases with a heat conduction coefficient $\chi<0.1 \rho^{2} v_{t h} / R$. These simulations can therefore be considered below the nonlinear threshold. 


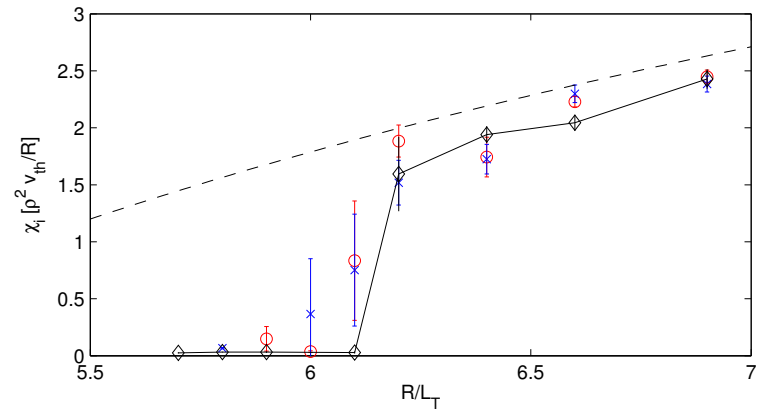

FIG. 3. Heat conduction coefficient in gyro-Bohm units $\left(\rho^{2} v_{t h} / R\right)$ as a function of the gradient length $R / L_{T}$ for the circular geometry and low dissipation / high resolution. The circles (colour red online) represent the case LH, the crosses (colour online blue) the case LS, and the diamonds connected with the solid line (colour online black) the case S6 of Table I. The dashed line represents the fit of Eq. (8)

\section{HIGH RESOLUTION / LOW DISSIPATION}

The investigation presented in the previous section show that at, or close to the non-linear threshold, the dissipation plays a role, which raises the question what threshold is obtained at very low dissipation.

Fig. 3 shows the heat conduction coefficient obtained with the circular geometry for two sets of simulations. Denoted with circles (red in the online version) are simulations obtained with a higher spatial resolution, both along the magnetic field as well as in the plane perpendicular to the magnetic field (case LH of Table I). Denoted with crosses (blue in the online version) is a case with a higher resolution in both the direction along the magnetic field as well as in the parallel velocity direction (case LS of Table I). The dashed lines represent the fits given by Eq. (8). The turbulence is simulated over a time interval 2400 (4800) $R / v_{t h}$ for the LH (LS) case, and the heat flux is averaged over a time interval [600 - 2400] ([1200 4800]). In both cases the higher resolution leads to a smaller dissipation when compared with the moderate resolution case of Fig. 1, which leads to a remarkable result: A non-linear threshold for the temperature gradient in the circular geometry case $R / L_{T c}=6.2$ is found, $30 \%$ larger than the value $\left(R / L_{\text {Tdim }}=4.7\right)$ obtained with a higher dissipation / lower resolution. Furthermore, the heat conduction coefficient does not go smoothly to zero, but rather has a finite value $\chi \approx 1.5$ at the non-linear threshold $[5,6]$.

Also in this case a finite heat flux, with rather large error bars, is obtained below the non-linear threshold $R / L_{T c}=$ 6.2. Although the averaged heat flux for $R / L_{T}=6.1$ is not negligible, quiet phases with a very low heat flux are again observed in this case. The temporal limited time interval of the subdued state is interpreted to result from the dissipative damping of the zonal mode. Therefore, the obtained heat fluxes below $R / L_{T}=6.2$ is considered non-physical.

If the interpretation adopted in this paper is correct, the results at higher resolution shown in Fig. 3 should also be obtained for the moderate resolution cases shown in Fig. 1, if the dissipation of the zonal mode is reduced at the same resolution. This has been verified by replacing the fourth order scheme for the convection along the field line with the sixth order scheme given by Eq. (4). The latter scheme is applied to the zonal mode $\left(k_{y}=0\right)$ only, while all other modes $\left(k_{y} \neq 0\right)$ are modelled using the fourth order scheme. Fig. 3 also shows (diamonds connected with a solid line, online colour black) the results of the simulations using this scheme (case S6 of Table I). Simulations have been run for 4800 normalized time units and for all cases in which no subdued transport is observed, the heat flux is averaged over a time interval [600 - 4800]. For the cases with subdued transport the time average is taken from the first time point at which a subdued state is observed, and the minimum time interval over which the heat flux is averaged is 720 normalized time units. Also for the 6th order scheme at moderate resolution a threshold value $R / L_{T c}=6.2$ is obtained, in agreement with the results of the $\mathrm{LH}$ and LS cases. Furthermore, the obtained values of $\chi$ for $R / L_{T}>6.2$ are in good agreement with the LH and LS cases, and around $15 \%$ smaller than the case S of Fig. 1 due to the stronger zonal flow. For the 6 th order scheme, $\left|\gamma^{(6)}(16)\right|<\left|\gamma^{(4)}(32)\right|$, and an almost zero heat flux is obtained for $R / L_{T}<6.2$. Finally, it is found that close to the threshold, rather long simulations are necessary to obtain the subdued turbulence state.

As mentioned above the closer $R / L_{T}$ is to the non-linear threshold, the longer it takes for the turbulence to be subdued. It can not be completely excluded that a higher threshold is obtained if the simulation time interval is extended. It has, however, been verified that a quenching does not occur in all cases, by extending the time interval for the case with $R / L_{T}=6.9$ (S6) to $15700 R / v_{t h}$. This excessive long run shows no sign of turbulence quenching.

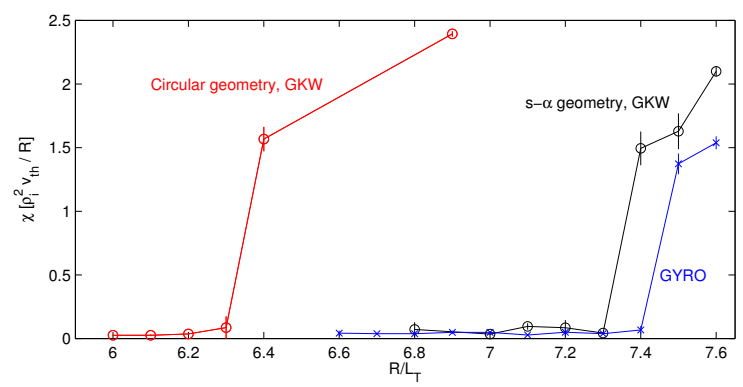

FIG. 4. Heat conduction coefficient in gyro-Bohm unites $\left(\rho^{2} v_{t h} / R\right)$ as a function of the gradient length $R / L_{T}$ for the $\hat{s}-\alpha$ and circular geometry (resolution case G6). For the $\hat{s}-\alpha$ geometry the results of GYRO (case GH) are also shown. 

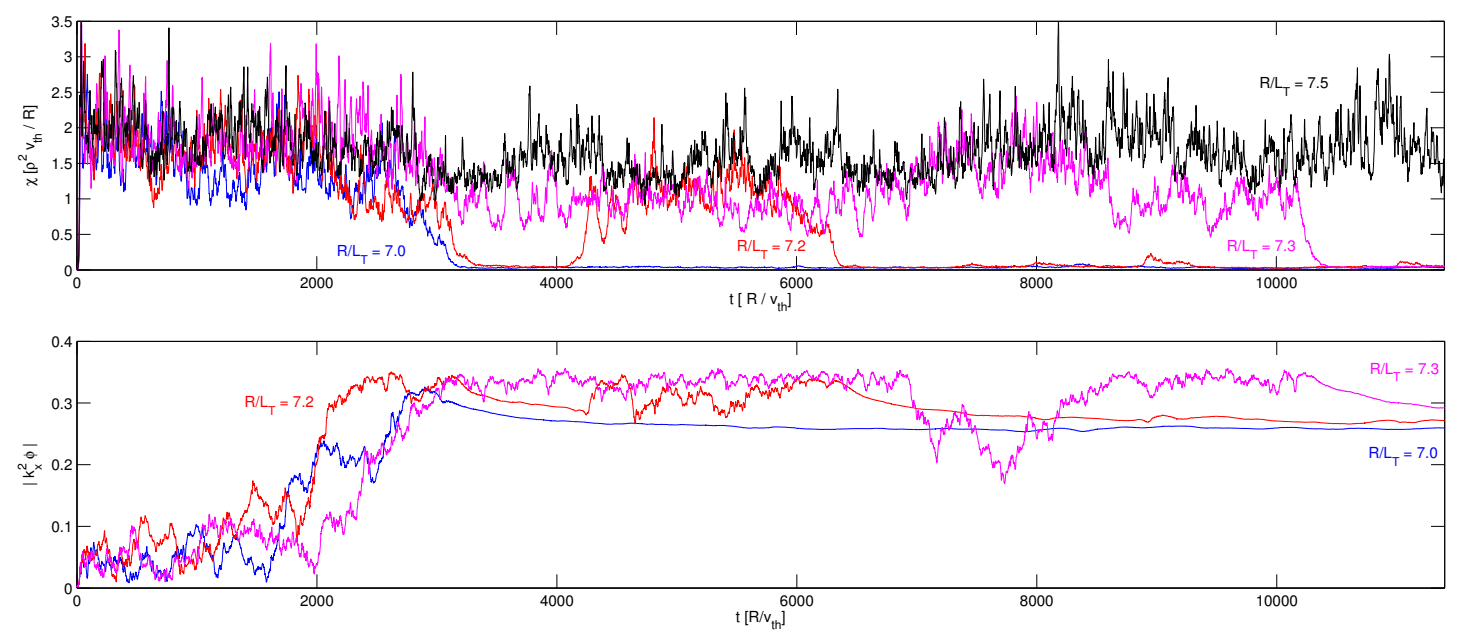

FIG. 5. Top: time traces of the heat conduction coefficient for $R / L_{T}=7.0$ (colour online blue), $R / L_{T}=7.2$ (colour online: red), $R / L_{T}=7.3$ (colour online magenta), and $R / L_{T}=7.5$ (colour online black) calculated with the resolution case G6 of Table I. Bottom: Time traces of $\omega_{E x B}^{\max }=\left(k_{x} \rho\right)^{2}|\phi|$ of the $k_{x} \rho=0.069$ mode, for three values of $R / L_{T}$ (online colours match that of the top graph).

\section{BENCHMARK}

A word of caution on the results of the previous section is in order. The results rely on rather long simulations with very little numerical dissipation at wave lengths much large than the grid size. A small numerical error in the transfer of energy could lead to a slow build up of the zonal mode, that can nevertheless reach high amplitudes due to the small dissipation. Indeed, it is possible that none of the numerous benchmarks performed with the code is sensitive to such a small error. The simulations using different dissipations settings in combination with the physical picture of the influence of the dissipation on the zonal flow give confidence to the results presented in the paper. However, they can not completely rule out the possibility of a small numerical error in the zonal flow drive. Therefore, a benchmark with the GYRO code [12] has been undertaken.

GYRO is particularly suited for this benchmark since its numerical implementation is quite different when compared with GKW. GYRO uses an orbit-grid to represent the perturbed distribution, and blending functions for the solution of the field equations. The representation of the velocity space, using pitch angle and energy, is quite different compared with GKW, which uses the parallel velocity and magnetic moment. Furthermore, no velocity space dissipation is applied in GYRO. Since GYRO uses a third order upwind scheme for the orbit integration (similar to the fourth order scheme described above), the numerical dissipation at fixed wavelengths can again be reduced through the increase of the number of orbit grid points. Simulations have been performed for the $\hat{s}-\alpha$ geometry and the cyclone base case parameters given above. Two resolutions for the orbit in- tegration $N_{s}=6$ (case GL) and $N_{s}=20$ (case GH) have been used. The number of blending functions was chosen equal to the number of orbit grid points in both cases. The GL case uses 4 passing pitch angle as well as 4 trapped pitch angle grid points, while the GH case uses 10 passing as well as 10 trapped grid points. All other parameters are the same in both cases: 128 radial grid points with $L_{x}=100 \rho$ (note that all quantities are given in GKW units), 24 toroidal modes with $k_{y}^{\max } \rho=1.48$, and 8 energy grid points. The resolution and box size in the plane perpendicular to the field are similar to that used in the simulations with GKW, but no special effort has been made to try and match these quantities exactly.

The simulation results of the GL case are included in Fig. 1 (crosses, online colour magenta). At this moderate resolution and limited time interval $\left(1900 R / v_{t h}\right)$ there is good agreement with the moderate resolution results obtained with GKW. Fig. 4 shows the comparison of the high resolution GYRO case $(\mathrm{GH})$ with the results of GKW. The latter have been obtained with a higher velocity space and parallel coordinate resolution (case G6 in Table I) when compared with the results of Fig. 3 in order to match the resolution in the GYRO case. The benchmark is performed for the $\hat{s}-\alpha$ geometry, but the GKW results for circular geometry are also shown. The GKW results have been obtained for a simulation interval $11400 R / v_{t h}$, with the $R / L_{T}=7.4$ case extended to $30000 R / v_{t h}$, while the smallest time interval of the GYRO runs was [0 1900] (for small $R / L_{T}$ where transport is quickly subdued) and the $R / L_{T}=7.5$ case has been simulated over a time interval $10400 R / v_{t h}$. The agreement between GKW and GYRO for the $\hat{s}-\alpha$ geometry is good, giving further confidence in the correctness of the results. There is a small difference in the thresh- 
old with $R / L_{T c}=7.4$ for GKW while $R / L_{T c}=7.5$ in the GYRO case. This might be related to the fact that GYRO has no velocity space dissipation, but if the threshold is set by a critical heat flux, it might also be due to the somewhat smaller heat fluxes obtained in the GYRO runs.

Also in the $\hat{s}-\alpha$ geometry a finite heat flux at a threshold $R / L_{T}=7.4$ is obtained, a value larger than that of the cyclone base case $R / L_{T}=6.9$. The results of these simulations, therefore, lead to the somewhat shocking conclusion that the cyclone base case, which uses the $\hat{s}-\alpha$ geometry, is non-linearly stable. The critical minimum heat conduction coefficient $\chi \approx 1.5$ is similar for both geometries. It suggests that a stationary turbulent state in a gradient driven simulation can only develop when the heat flux, heat conductivity, or turbulent intensity exceeds a critical value.

\section{ZONAL FLOW EVOLUTION}

The previous sections show that low dissipation as well as a sufficiently long time interval are necessary to obtain the correct heat flux in collisionless ITG turbulence. Furthermore, the zonal ExB shear plays an essential role in obtaining the finite heat flux threshold. The reason behind the long time scales involved, however, remains to be clarified.

Fig. 5 shows the time traces of the heat conduction coefficient, obtained in the $\hat{s}-\alpha$ geometry with resolution G6 of Table. I, for $R / L_{T}=7.0,7.2,7.3$, and 7.5, as a function of the normalized time. Several observations can be made. First, as mentioned above, the time point at which turbulence is subdued, increases when $R / L_{T}$ is closer to the threshold. Second, also for gradient lengths above the threshold, the heat flux shows an evolution on long time scales, with the averaged heat flux smaller at later times. Fig. 5 also shows the potential (normalized with $e /\left(\rho_{*} T_{e}\right)$ with $\left.\rho_{*}=\rho / R\right)$ times the wave vector squared $\left(k_{x} \rho\right)^{2}\left|\phi\left(0, k_{x}\right)\right|$, for the $k_{x} \rho=0.069$ zonal mode as a function of time. This quantity is a measure of the shearing rate $\left(\omega_{E x B}^{\max }\right)$ obtained at the radial position where the potential of the long wave length zonal mode reaches its maximum value. The $k_{x} \rho=0.069$ mode is the longest wavelength zonal mode in the computational domain, and dominates in amplitude, by an order of magnitude, at late times in the evolution. Even before turbulence is subdued, a clear correlation between the amplitude of this mode and the magnitude of the heat transport is observed, and turbulence is subdued only when a specific amplitude of this mode is reached.

The long wavelength zonal flow generation is likely related to the modulation instability $[15,16]$. Indeed the growth rate of the modulation instability scales with the wave vector of the zonal mode and, therefore, is relatively small for these long wavelength modes. It is noted, however, that the amplitude of the zonal mode as a function of time does not reflect an exponential growth, and can be, especially at higher gradient lengths, a nonmonotonic function of time. Estimating the typical timescale of growth using $\tau=2(t(0.3)-t(0.18))$, where $t(\beta)$ is the first time point for which the amplitude of the mode reaches $\beta$, one obtains time intervals $\tau=344,1800$, and 1000 for $R / L_{T}=7.0,7.2$, and 7.3, whereas the estimated growth rate of the modulation instability is $\gamma_{M} \approx 0.01$. Furthermore, when increasing $R / L_{T}$ the time-scale on which the long wave length zonal flow develops appears to be longer, a result that is not easily explained on the basis of the modulation instability alone. Although it is likely that the zonal mode grows through a modulation instability, it is also clear that additional physics is involved, perhaps not surprisingly in a fully developed turbulent state. The long time scales involved do however, explain the sensitivity to dissipation as well as the long time intervals required for the simulations.

Since the zonal mode involved in the quenching is the longest wavelength in the box one could argue that increasing the box size could change the results. The simulations with the large computational domain (case LH of Table I), however, yield similar results for the threshold and heat fluxes. In the latter case the zonal mode responsible for the quenching have a wavelength equal to only half the box size.
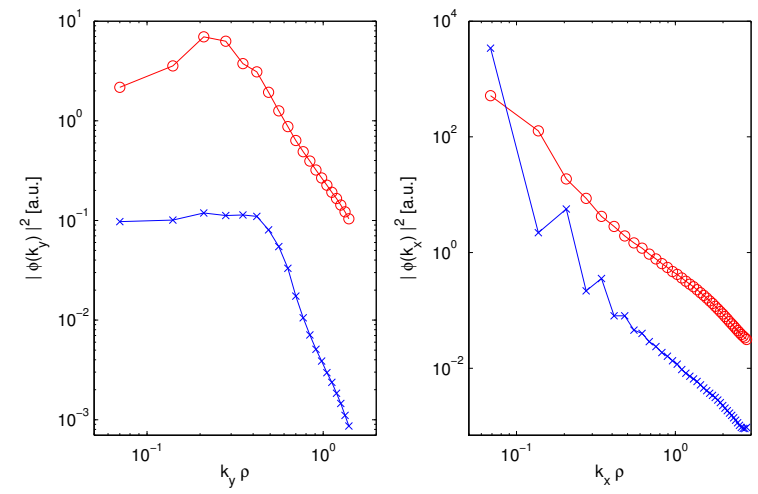

FIG. 6. Spectra $\left|\phi\left(k_{y}\right)\right|^{2}=\sum_{k_{x}}\left|\phi\left(k_{x}, k_{y}\right)\right|^{2}$ and $\left|\phi\left(k_{x}\right)\right|^{2}=$ $\sum_{k_{y}}\left|\phi\left(k_{x}, k_{y}\right)\right|^{2}$ of the simulation with $R / L_{T}=7.2$ (case G6 of Table I) as a function of $k_{y} \rho$ and $k_{x} \rho$, respectively. Circles (colour online red) represent the average over the time interval $[500,2000]$, whereas crosses (colour online blue) represent the average of the time interval [7000, 11000].

After turbulence is subdued the zonal flow slowly decays, in agreement with the damping through dissipation. For $R / L_{T}=7.0$ and 7.2 , however, it is observed that it saturates at a finite value, which for $R / L_{T}=7.0$ is maintained over a time interval of 7000 normalized time units. In this, and all other cases, the heat flux and turbulent transport are not completely quenched. Turbulence is subdued, with the heat flux two orders of mag- 
nitude lower, rather than completely quenched. Fig. 6 compares the spectra for $R / L_{T}=7.2$ averaged over the time interval [500, 2000] (fully developed turbulence) and [7000, 11000] (subdued turbulence). It can be seen that also in the latter case, the spectrum has a shape similar to that of the fully developed turbulence, albeit strongly reduced in magnitude. It appears that in the subdued state the low level turbulence can still drive the zonal flow against the dissipative damping. Only closer to the threshold, the evolution of the heat flux is similar to the observations in Ref. [10], which investigates the heat flux below the nonlinear threshold connected with the collisional damping of the zonal flow. In the latter case the zonal flow decays up to a point where a substantial heat flux reappears.

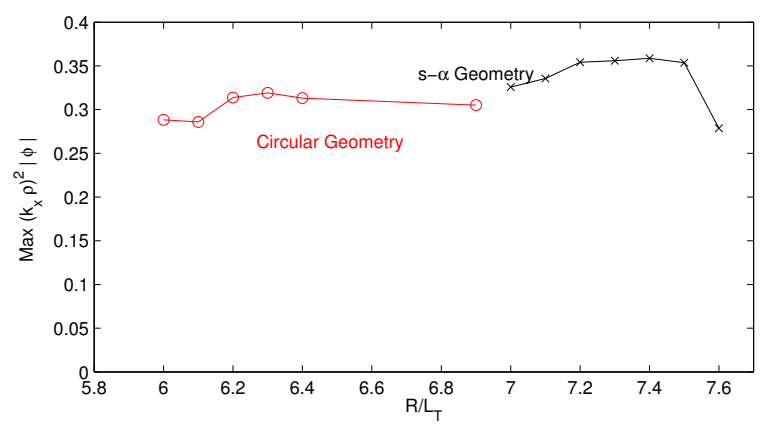

FIG. 7. Maximum values of $\left(k_{x} \rho\right)^{2}|\phi|$ obtained in the time trace of the simulations as a function of the temperature gradient $R / L_{T}$. Circles (colour online red) represent the circular geometry, while crosses (colour online black) represent the $\hat{s}-\alpha$ geometry.

Another striking observation can be made in Fig. 5. The amplitude of the long wave length zonal flow appears to be limited to a particular value, and turbulence is not immediately subdued when this value is reached. For $R / L_{T}=7.3$, for instance, the long wave length zonal flow has a nearly constant maximum value for a time interval of 4000 normalized time units. During this time, turbulence is reduced compared to the time intervals in which the long wave length zonal mode has a much smaller amplitude, but it is not subdued. The limitation of the long wave length zonal flow amplitude is observed in all simulations, and the maximum value is relatively independent of the temperature gradient as shown in Fig. 7, which gives the maximum value of $\left(k_{x} \rho\right)^{2}|\phi|$ obtained in a time trace as a function of $R / L_{T}$. The limitation of the zonal flow amplitude is possibly related to a threshold behaviour for the Kelvin Helmholtz instability [17], although this could not be verified within the scope of this paper. A maximum amplitude of the long wavelength zonal flow corresponds to a maximum shearing rate, and one may argue that it explains a threshold behaviour since at sufficient high temperature gradients the growth rate of the ITG will exceed the shearing rate
$\gamma>\omega_{E x B}^{\max }$, and turbulence can no longer be subdued through ExB shearing. However, the physics picture appears to be more complicated. First it is noted, that the mechanism above does not explain the finite heat flux at the threshold. The effect of the shearing rate is usually modelled as generating an offset for the growth rate, i.e. one expects the heat conduction coefficient to scale as $\chi \propto\left(\gamma-\left|\omega_{E x B}\right|\right)$. This model can, therefore, explain an up-shift in the nonlinear threshold, but not a finite heat flux at the threshold. Second, the condition $\gamma=\omega_{E x B}^{\max }$ is also satisfied for gradient lengths above the threshold as will be shown below.

Fig. 8 gives the shearing rate $\omega_{E x B}$ for the simulation with $R / L_{T}=7.2$ as a function of the radial coordinate for different time intervals. The thick line gives $\omega_{E x B}$ averaged over the time interval [500 2000] which represents the initial state, the time interval [4500 6000] which represents a reduced turbulence state, and [7000 10000] in which the turbulence is subdued. The dashed line in the plots gives the (instantaneous) shearing rate at the middle of the chosen time interval, and the dash-dotted line (online colour blue) gives the growth rate $(\gamma)$ of the most unstable mode $\left(k_{y} \rho=0.4\right)$. These three time intervals represent the three states of turbulence that are observed in the various simulations. In the initial state ([500 2000]) the averaged shearing rate is relatively small and shows no clear structure. In this case the turbulence is not reduced with respect to the high dissipation results. For the reduced turbulence state ([4500 6000]) the long wave length zonal flow has reached its maximum amplitude, and transport is reduced but not subdued. The ExB shearing rate can be observed to reach values equal to or larger than the growth rate over a large part of the radial domain. Furthermore, a radial structure can be observed to exist, with the shearing rate roughly equal to $\pm \gamma$ over a large part of the radial domain, with one relatively steep transition $\omega_{E x B}=-\gamma \rightarrow+\gamma$, and a less steep transition $\omega_{E x B}=+\gamma \rightarrow-\gamma$ (note that the radial boundary conditions are periodic). This structure has been observed previously in global GISELA simulations, and is referred to as staircase [21]. Note that the fluctuations in the ExB shearing are larger than the averaged ExB shearing rate, as indicated by the dashed line. Finally, the interval [7000 10000] represents the state in which the transport is subdued. In this case the shearing rate at nearly all radial positions is equal to $\pm \gamma$, with very steep transitions from positive to negative and vice versa. We will, therefore, refer to this state as a 'fully developed staircase'. In all investigated states of subdued transport a fully developed staircase is observed. Indeed, only for a fully developed staircase is the shearing rate equal to the growth rate over (almost) the entire radial domain, and can the ExB shearing suppress the turbulence at all radial locations. For the fully developed staircase the fluctuations in the shearing rate are small compared with the time averaged shearing rate as shown by the dashed line. Note, furthermore, that the 

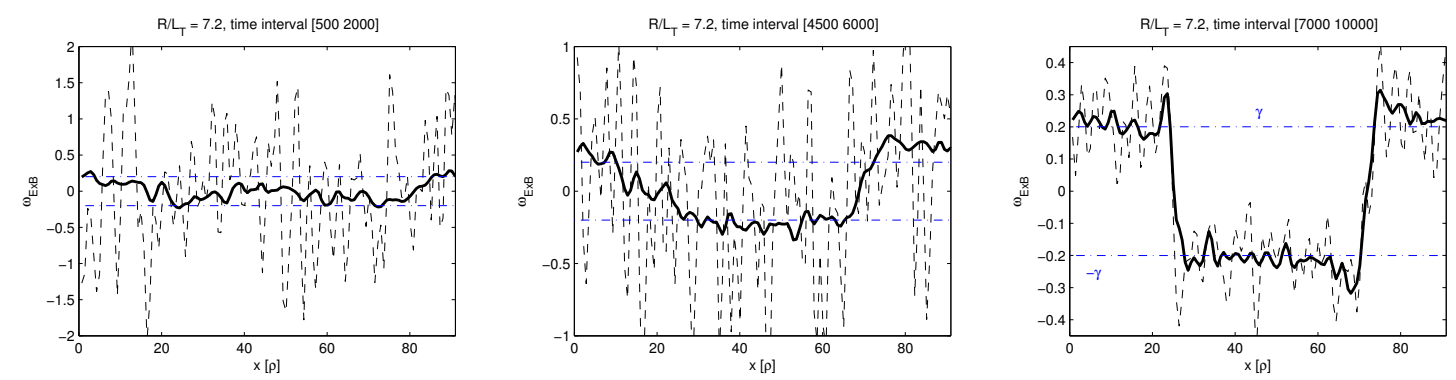

FIG. 8. The shearing rate $\omega_{E x B}=\partial^{2} \phi / \partial x^{2} /(2 B)$ as a function of the radial coordinate $(x)$ normalized with the Larmor radius for different time intervals of $R / L_{T}=7.2$ case with $\hat{s}-\alpha$ geometry. The thick line gives the shearing rate averaged over the time interval given in the title of the figure, while the dashed line gives the shearing rate at a time-point in the centre of the interval. The dash-dotted line (online colour blue) gives the growth rate $\pm \gamma$ of the most unstable mode.

radial structure formed in the potential / shearing rate is reflected in the shape of the spectrum $\sum_{k_{y}}\left|\phi\left(k_{x}, k_{y}\right)\right|^{2}$ shown in Fig. 6

The reduced turbulence state with staircase formation is also observed above the threshold as shown in Fig. 9. Different from the $R / L_{T}=7.2$ case, where the reduced turbulence state is only a quasi-stationary state, the staircase is observed to exist for a time interval $>5000$ normalized time units in the $R / L_{T}=7.4$ case, and is assumed to be a stationary state here. Also for $R / L_{T}=7.4$ the time averaged shearing rate is equal to the growth rate over a large part of the radial domain. The time averaged heat conduction coefficient, however, shows little radial structure, as indeed it must, since a stationary state demands a uniform radial heat flux. The reason for the finite heat flux in the radial domain with $\gamma=\left|\omega_{E x B}\right|$ is the occurrence of avalanches as shown in Fig. 9, which shows the heat conduction coefficient as a function of the time and the radial coordinate. Avalanches have been observed in turbulent simulations under various circumstances [18-22], and the behaviour here is similar to the one previously reported in connection with staircases [21]. Avalanches start in the region $\left|\omega_{E x B}\right|<\gamma$ and propagate outward / inward across the staircase. They allow for a finite heat flux in the regions where $\gamma=\left|\omega_{E x B}\right|$, despite the marginal stability of the turbulence in these regions. This conclusion is supported by the observed perturbations in the temperature gradient also shown in Fig. 9. These perturbations are correlated with the observed perturbations in the heat conduction, and have a magnitude sufficient to generate local growth rates well above the averaged shearing rate.

From the observations described above the following physical picture of the finite heat flux threshold emerges. Turbulence is subdued only when the staircase structure fully develops. This occurs below the threshold in the temperature gradient. Above the threshold the staircase does not develop fully and a finite heat flux occurs which is supported by avalanches propagating through the regions of marginal stability $\left(\gamma=\omega_{E x B}\right)$. Of course, it remains to explain under what circumstances the staircase can develop fully and, in particular, what the influence of the temperature gradient on its development is. These questions are beyond the scope of this paper.

\section{CONCLUSION}

In this paper, gradient driven ion temperature gradient turbulence close to the non-linear threshold has been investigated in detail. It is shown that a long time-scale evolution in the heat flux occurs, provided the dissipation is sufficiently small. A finite dissipative damping of the zonal mode, can maintain a finite heat flux, in disagreement with the collisionless ITG turbulence model. At sufficiently small dissipation it is found that the threshold for turbulence generation is considerably larger ( $30 \%)$ compared with the threshold predicted by extrapolating the heat flux to zero. Furthermore, the heat flux does not go smoothly to zero when approached from above, but rather has a finite value at the threshold, below which no stationary fully developed turbulent state exists [6].

The long time scale in the evolution of the heat flux, with smaller heat fluxes at later times, is shown to be connected with a slowly growing long wave length zonal flow. The amplitude of the long wave length electro-static potential is found to be limited to a specific value which shows no strong dependence on the temperature gradient length or the turbulence intensity. At the maximum amplitude, turbulence reduction is observed for gradient lengths close to the threshold, but turbulence is not subdued in all cases, or occurs only after a considerable time interval. The time averaged potential shows a staircase structure, with the ExB shearing rate comparable to the growth rate over a large range of the radial domain. The latter condition suggests that the ITG mode is marginally stable in these regions. However, a finite heat flux is generated over the entire radial domain through the occurrence of avalanches which emerge from the region with $\left|\omega_{E x B}\right|<\gamma$ and propagate through the regions with $\left|\omega_{E x B}\right|=\gamma$. Turbulence is subdued only if the staircase is fully developed with $\left|\omega_{E x B}\right|=\gamma$ over (almost) the 


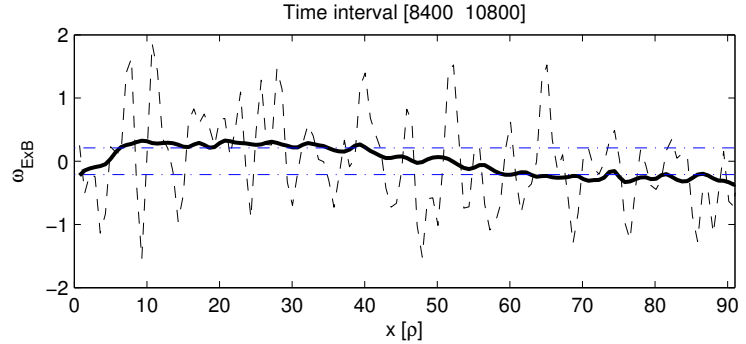

$\chi\left[\rho^{2} v_{t h} / R\right]$

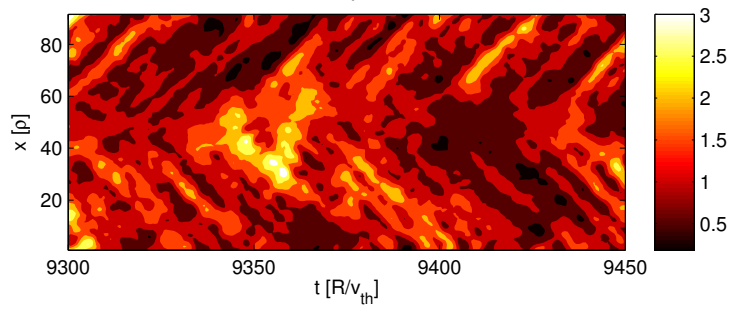

$\delta\left(\mathrm{R} / \mathrm{L}_{\mathrm{T}}\right)$

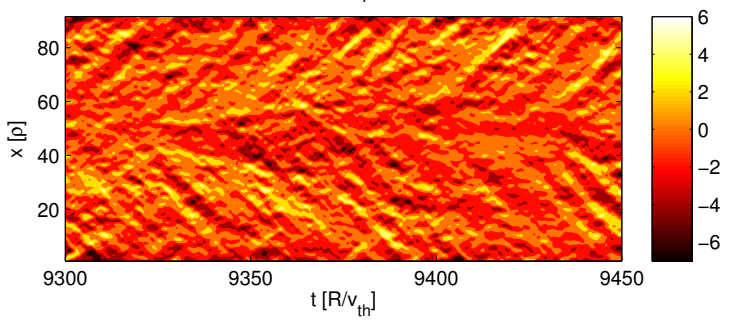

FIG. 9. Top: Shearing rate $\omega_{E x B}$ at $t=9600$ (dashed line) as well as averaged over the time interval [8400 10800] (solid thick line) as a function of the radial coordinate $(x)$ for $R / L_{T}=7.4$ and $\hat{s}-\alpha$ geometry. The dash dotted line (colour online blue) gives the growth rate $( \pm \gamma)$ of the ITG. Middle: Contour plot of the heat conduction coefficient as a function of time and radial coordinate. Bottom: Contour plot of the radial temperature gradient due to the turbulent temperature fluctuations as a function of time and radial coordinate. entire radial domain. Above the nonlinear threshold in the temperature gradient $R / L_{T c}$, the staircase can no longer fully develop and a finite heat flux is generated through the occurrence of avalanches.

A reduced model, using adiabatic electrons and neglecting collisions, has been used for the studies in this paper. Although, in the view of the authors, it is important to understand this basic case, collisions can be expected to damp the zonal flow, and alter the picture at sufficiently high collision frequencies. For many current experiments, and certainly for a reactor, the collisional damping however is smaller than the damping generated by the numerical dissipation at moderate resolution. Although collisions will likely allow for a finite heat flux below the collisionless threshold, it can be expected that a steep increase in the heat flux is observed close to the collisionless threshold in a sufficiently hot plasma, while above the threshold collisions have little effect. Besides the neglect of the collisions, also the use of adiabatic electrons present a simplification when compared with an experimentally relevant case. Zonal flows are weaker in the case of kinetic electrons. They, however, still regulate the transport, and it can be expected that the physics model discussed in this paper also applies to the case of kinetic electrons, although the effects are perhaps less pronounced.

The results presented in this paper show that the determination of the nonlinear threshold requires a careful approach. In the collisionless case, the finite heat flux at the nonlinear threshold in the gradient driven case does not allow to determine the gradient length for which the averaged heat flux goes to zero, and a flux driven approach must be used to obtain this gradient length $[5,6]$. Although it is unclear how collisions or kinetic electrons change this picture precisely, the results do show that a proper determination of the heat flux requires the dissipative damping to be small compared with the collisional damping, and that in the case of small collisionality, relatively long simulations may be required.
[1] A.M. Dimits, G. Bateman, M.A. Beer, B.I. Cohen, W. Dorland, G.W. Hammett, C. Kim, J.E. Kinsey, M. Kotschenreuther, A.H. Kritz, L.L. Lao, J. Mandrekas, W.M. Nevins, S.E. Parker, A.J. Redd, D.E. Shumaker, R. Sydora, J. Weiland, Phys. Plasmas 7, 969 (2000)

[2] A. Hasegawa, C.G. Maclennan, and Y. Kodama, Phys. Fluids 22, 2122, (1979)

[3] R.E. Waltz, G.D. Kerbel, J. Milovich, Phys. Plasmas 1, 2229 (1994).

[4] P.H. Diamond, S.-I. Itoh, K. Itoh, and T.S. Hahm, Plasma Phys. Control. Fusion 47, R35 (2005)

[5] F. Rath, A.G. Peeters, R. Buchholz, S.R. Grosshauser, P. Migliano, D. Strintzi, and A. Weikl, On the nonlinear upshift of the temperature gradient threshold in ion temperature gradient turbulence, submitted to Nuclear
Fusion.

[6] F. Rath, A.G. Peeters, R. Buchholz, S.R. Grosshauser, P. Migliano, D. Strintzi, and A. Weikl, Phys. Plasmas 23, 052309 (2016)

[7] A.G. Peeters, Y. Camenen, F.J. Casson, W.A. Hornsby, A.P. Snodin, D. Strintzi, G. Szepesi, Comp. Phys. Comm. 180, 2650 (2009)

[8] J.A. Krommes, G. Hu, Phys. Plasmas 1, 3211 (1994).

[9] J. Candy, R.E. Waltz, Phys. Plasmas 13, 032310 (2006)

[10] Z. Lin, T.S. Hahm, W.W. Lee, W.M. Tang, P.H. Diamond, Phys. Rev. Lett. 83, 3645 (1999)

[11] Y. Xiao, P.J. Catto, K. Molvig, Phys. Plasma 14, 032302 (2007)

[12] J. Candy, R.E. Waltz, Journal Comp. Phys. 186, 545 (2003) 
[13] X. Lapillonne, S. Brunner, T. Dannert, S. Jolliet, A. Marinoni, L. Villard, T. Görler, F. Jenko, F. Merz, Phys. Plasmas 16, 032308 (2009)

[14] G.L. Falchetto, B.D. Scott, P. Angelino, A. Bottino, T. Dannert, V. Grandgirard, S. Janhunen, F. Jenko, S. Jolliet, A. Kendl, B.F. McMillan, V. Naulin, A.H. Nielsen, M. Ottaviani, A.G. Peeters, M.J. Pueschel, D. Reiser, T.T. Ribeiro, and M. Romanelli, Plasma Phys. Control. Fusion 50, 124015 (2008)

[15] A.I. Smolyakov, P.H. Diamond, A.I. Shevchenko, Phys. Plasmas 7, 1349 (2000)

[16] L. Chen, Z. Lin, R. White, Phys. Plasmas 7, 3129 (2000)
[17] B.N. Rogers, W. Dorland, M. Kotschenreuther, Phys. Rev. Lett. 855336 (2000)

[18] X. Garbet, R.E. Waltz, Phys. Plasmas 5, 2836 (1998)

[19] Y. Sarazin, X. Garbet, Ph. Ghendrih, S. Benkadda, Phys. Plasmas 7, 1085 (2000)

[20] B.F. McMillan, S. Jolliet, T.M. Tran, L. Villard, A. Bottino, P. Angelino, Phys. Plasmas 16, 022310 (2009)

[21] G. Dif-Pradalier, P.H. Diamond, V. Grandgirard, Y. Sarazin, J. Abiteboul, X. Garbet, Ph. Ghendrih, A. Strugarek, S. Ku, and C.S. Chang, Phys. Rev. E 82, 025401 (2010).

[22] T. Görler, X. Lapillonne, S. Brunner, T. Dannert, F. Jenko, S.K. Afhdam, Phys. Plasmas 18056103 (2011) 